Check for updates

Cite this: Mater. Adv., 2020 1,2107

Received 4th July 2020

Accepted 12th August 2020

DOI: 10.1039/d0ma00478b

rsc.li/materials-advances

\section{Improvement of the strength of poly(acrylic acid) hydrogels by the incorporation of functionally modified nanocrystalline Cellulose}

\author{
Md. Amzad Hossain, (D) ${ }^{a}$ Chanchal Kumar Roy, (D) *a Stephen Don Sarkar, (D) ${ }^{a}$ \\ Hridoy Roy, (D) ${ }^{b}$ A. Hasan Howlader (D) ac and Shakhawat H. Firoz (D) *a
}

\begin{abstract}
Finding different strategies to incorporate functionalized nanocrystalline cellulose (NCC) into polymeric materials is a fascinating domain of current research. In this study, dialdehyde nanocrystalline cellulose (DANC) and dicarboxylated nanocrystalline cellulose (DCNC) were prepared by the functionalization of NCC at the C-2 and C-3 positions of the glucose moiety by a selective chemical oxidation process. The functionalized NCCs were successfully incorporated into poly(acrylic acid) (PAA) hydrogels to improve their mechanical properties. The PAA hydrogel incorporated with DCNC (PAA-DCNC) showed the most significant enhancement in mechanical properties compared to the neat PAA, PAA-NCC, and PAA-DANC hydrogels. The Young's modulus of the neat PAA hydrogel was only $90.2 \mathrm{kPa}$, which was increased by about six times $(619.1 \mathrm{kPa})$ by the addition of a minimal amount $(0.058 \mathrm{wt} \%)$ of DCNC with $40 \%$ carboxylation (DCNC-40). Six times enhancement in the tensile strength was observed in the presence of DCNC-40. The toughness of PAA-DCNC-40 hydrogel was $522.2 \mathrm{~kJ} \mathrm{~m}^{-3}$, which was only $97.4 \mathrm{~kJ} \mathrm{~m}^{-3}$ in the absence of DCNC. Mechanical and water swelling properties of the prepared hydrogels were examined to understand the mechanism of interaction for the enhancement of performance.
\end{abstract}

\section{Introduction}

Hydrogels, with their extraordinary soft-wet nature and high-water retention capacity, are one of the most promising materials for biochemical applications. ${ }^{1-4}$ They have been used in bio-sensing, ${ }^{5}$ bio-separation, ${ }^{6}$ and drug-delivery systems. ${ }^{7}$ However, the wide range of applications of the hydrogels is still limited, particularly in the field of tissue engineering due to their weak mechanical properties. Poor mechanical performance is frequently associated with the insufficient physical, chemical, or mechanical bonding of polymer chains present in the material. Incorporation of synthetic crosslinkers like $N, N^{\prime}$-methylenebisacrylamide (MBA) for the formation of an integrated polymer network by making numerous covalent crosslinking points is a general trend to improve the mechanical properties of hydrogels. Besides, reinforcing strategies by the incorporation of functionalized nanomaterials as additives or filler materials such as clays, ${ }^{8}$ silica nanoparticles, ${ }^{9}$ carbon nanotubes, ${ }^{10}$ ferritin particles, ${ }^{11}$ and graphene oxide, ${ }^{12}$ etc. to

\footnotetext{
${ }^{a}$ Department of Chemistry, Bangladesh University of Engineering and Technology, Dhaka 1000, Bangladesh. E-mail: ckroy@chem.buet.ac.bd, shfiroz@chem.buet.ac.bd

${ }^{b}$ Department of Chemical Engineering, Bangladesh University of Engineering and Technology, Dhaka 1000, Bangladesh

${ }^{c}$ Department of Chemistry and Biochemistry, Florida International University, Miami, FL 33199, USA
}

prepare nanocomposite hydrogels have also attracted great attention due to the easy fabrication process. But these processes often result in non-biodegradability and are toxic to the human body. Here, natural organic-based fillers and crosslinkers are promising materials to overcome these limitations. In biomedical applications, there is an appeal to using hydrogels with natural components, which could ensure a long-term non-toxic effect in the living body. ${ }^{13,14}$ Among them, nanocrystalline cellulose (NCC) has attracted considerable attention due to its unique properties such as crystalline nature, high mechanical strength but lightweight, and most importantly biodegradability and biocompatibility. ${ }^{15-18}$ Enhancement of the mechanical properties of various kinds of hydrogels through the incorporation of natural fibers like NCC will certainly improve the biodegradability and biocompatibility of the material, and expand the practical application of hydrogels in biomedical engineering especially for the fabrication of artificial cartilage and biological organs.

NCC has been prepared from various renewable cellulose sources such as rice husk, ${ }^{19}$ cotton, ${ }^{20}$ wood, ${ }^{21}$ algae, ${ }^{22}$ etc. either by hydrolysis ${ }^{23}$ or oxidation, ${ }^{24}$ yielding nanocrystals with different surface functionalities. Enhancements in mechanical performance have been investigated by the incorporation of NCC in natural polymeric materials like starch, ${ }^{25}$ chitosan, ${ }^{26,27}$ etc. It has also been applied in synthetic polymeric materials such as poly(vinyl alcohol), ${ }^{28}$ polyacrylamide, ${ }^{29,30}$ poly(acrylic 
acid), ${ }^{31,32}$ polyethylene glycol, ${ }^{33}$ etc. The incorporation of NCC introduces an intermolecular bonding in a polymer matrix, which eventually improves the compactness of the hydrogel. But this interaction is limited to some specific polymeric backbones, and the bonding strength is weak. However, chemical modification of NCC demonstrated improved mechanical performances of different hydrogels through strengthening of bonding. ${ }^{3-36}$ A series of works to functionalize NCC have been conducted where the surface of NCC was grafted with different functional groups like acetyl, silyl, aldehyde, carboxylate, amine, hydrazine, etc. to improve its functionality. ${ }^{37-39}$ Among these, carboxylate functionalization of NCC has attracted considerable attention for its inherent water soluble forms which are compatible with polymer matrices. The incorporation of carboxylate functionalized NCC improved the mechanical strength of hydrogels by the formation of intermolecular hydrogen bonds. ${ }^{40}$ Carboxylate functionalized NCCs are conventionally obtained by the most common TEMPO oxidation of the primary hydroxyl group at the C-6 position of glucose. ${ }^{18,41}$ This functionalization generates only one carboxylate group per glucose unit on the NCC surface. Thus, it results in a small number of interacting sites for bonding. Here, increasing the number of interacting sites will improve the capacity of NCC to enhance the mechanical strength of hydrogels.

Meanwhile, Liimatainen et al. reported a sequential oxidation method using periodate and chlorite to oxidize vicinal hydroxyl groups of cellulose at the C-2 and C-3 positions of the glucose moiety to introduce dicarboxylate groups. ${ }^{42}$ Due to the formation of two carboxyl groups in each accessible glucose unit, the number of interacting sites will be increased and the reinforcing capability of NCC will be enhanced. The functionalization takes place through the formation of aldehyde groups and which are finally oxidized to carboxylic groups, which gives the flexibility to introduce different functionalities. Additionally, the extent of such functionalization can be controlled by setting up the reaction conditions, and so various functionally modified cellulose can be prepared. Nevertheless, for the chain opening of the glucose unit, this type of functionalized cellulose is expected to incur conformational changes in the polymer of NCC. To the best of our knowledge, the behavior of such functionalized cellulose for the improvement in the properties of synthetic hydrogels has not been explored yet.

Herein, we prepared poly(acrylic acid) (PAA) hydrogels reinforced with 2,3-dicarboxylic nanocrystalline cellulose (DCNC) and 2,3-dialdehyde nanocrystalline cellulose (DANC) for the improvement of the mechanical performance. A systematic study of the mechanical test and swelling test of the DCNC incorporated hydrogel was conducted. The participation and contribution of DCNC in the course of mechanical behavior were investigated by comparing the properties with neat PAA hydrogel, as well as with PAA hydrogels containing NCC and DANC as filler materials. Here, a comprehensive study of the preparation, characterization, and investigation of PAA hydrogel incorporated with different functional NCCs has been presented, which would significantly broaden the application of the materials.

\section{Experimental}

\subsection{Chemicals and materials}

Microcrystalline cellulose (MCC), sodium metaperiodate $\left(\mathrm{NaIO}_{4}\right)$, hydroxylamine hydrochloride $\left(\mathrm{NH}_{2} \mathrm{OH} \cdot \mathrm{HCl}\right)$ and $N, N^{\prime}$-methelynebisacrylamide (MBA) were purchased from Sigma-Aldrich. Ethylene glycol $\left(\mathrm{C}_{2} \mathrm{H}_{6} \mathrm{O}_{2}\right)$, sodium chloride $(\mathrm{NaCl})$, sodium chlorite $\left(\mathrm{NaClO}_{2}\right)$, hydrogen peroxide $\left(\mathrm{H}_{2} \mathrm{O}_{2}\right)$, ethanol $\left(\mathrm{C}_{2} \mathrm{H}_{5} \mathrm{OH}\right)$, potassium hydrogen phthalate, potassium dihydrogen phosphate $\left(\mathrm{KH}_{2} \mathrm{PO}_{4}\right)$, hydrochloric acid $(\mathrm{HCl})$ and sodium hydroxide $(\mathrm{NaOH})$ were procured from Merck, Germany. Acrylic acid (AA) and ammonium persulfate (APS) were purchased from BDH Chemicals. Sulfuric acid was obtained from RCI Labscan Ltd., Thailand. All the chemicals were analytical reagent grade and used as received. Cellulose membrane dialysis tubing (molecular weight cut-off 12000-14000) was purchased from Sigma-Aldrich, Germany. Aqueous solutions of all the experiments were prepared using deionized (DI) water.

\subsection{Preparation of NCC from MCC}

$5.0 \mathrm{~g}$ of MCC was mixed with a solution of $100 \mathrm{~mL} \mathrm{63.5 \%} \mathrm{(w/w)}$ of $\mathrm{H}_{2} \mathrm{SO}_{4}$ in a round bottom flask. The mixture was hydrolyzed at $60{ }^{\circ} \mathrm{C}$ for $90 \mathrm{~min}$ with continuous magnetic stirring. The reaction was quenched by adding ten-fold DI water, and ultrasonication was applied for $10 \mathrm{~min}$. The resulting suspension was allowed to settle down for $4 \mathrm{~h}$ after ultrasonication. The mixture was separated into two layers consisting of a transparent top layer of water. The top layer was decanted off, and the process of washing was continued until a milky white suspension was formed. The dispersed suspension was then washed by adding DI water with repeated centrifugation at $6000 \mathrm{rpm}$, which removed the excess acid and other watersoluble fragments. It was continued until a constant $\mathrm{pH}$ of 4 for the filtrate was obtained. NCC was dialyzed against DI water with dialysis tubes until the $\mathrm{pH}$ and conductivity became steady. Finally, the purified NCC suspension was kept in a refrigerator at $5{ }^{\circ} \mathrm{C}$ and used for further characterization and functionalization.

\subsection{Preparation of DANC from NCC}

The selective oxidation of the glucose moiety of NCC was performed to obtain DANC. For that process, $13.38 \mathrm{~g}$ of $\mathrm{NaIO}_{4}$ was added to $500 \mathrm{~mL}$ aqueous suspension of $2 \%(\mathrm{w} / \mathrm{v})$ NCC in a round bottom flask and stirred at $48{ }^{\circ} \mathrm{C}$ in an oil bath for $20 \mathrm{~h}$ in the absence of light. An excess amount of ethylene glycol (equivalent to $\mathrm{NaIO}_{4}$ ) was added to the mixture to quench the reaction, and a viscous suspension of DANC was obtained. It was washed with DI water and separated by repeated centrifugation. The product was dialyzed for several days with dialysis tubes against DI water to get a constant conductivity. A fraction of the DANC suspension was vacuum dried for characterization, another portion was used for preparing DCNC, and the remaining part was stored in aqueous suspension form in a refrigerator at $5{ }^{\circ} \mathrm{C}$. The aldehyde content (AC) of DANC was determined following the oxime formation method reported by Veelaert et al. and was found to be $40 \% .{ }^{43}$ For this determination, $0.3 \mathrm{~g}$ of DANC (from $0.04 \mathrm{~g} \mathrm{~mL}^{-1}$ aqueous 
suspension) was taken in a $250 \mathrm{~mL}$ beaker and the $\mathrm{pH}$ was adjusted to 5 with $\mathrm{HCl}$. The $\mathrm{pH}$ of a $20 \mathrm{~mL}$ hydroxylamine hydrochloride solution $(5 \%, \mathrm{w} / \mathrm{w})$ was also adjusted to 5 separately by adding $0.1 \mathrm{M} \mathrm{NaOH}$. Here, the volume consumption of $\mathrm{NaOH}$ solution was recorded as $V_{\mathrm{c}}(\mathrm{L})$. The same concentration of NCC suspension at $\mathrm{pH} 5$ was used as a blank and its volume consumption of the alkali solution was recorded as $V_{\mathrm{b}}(\mathrm{L})$. The $\mathrm{AC}$ in DANC was calculated using the following equation,

$$
\mathrm{AC}=\frac{M_{\mathrm{NaOH}} \times\left(V_{\mathrm{c}}-V_{\mathrm{b}}\right) \times 162}{m \times 1000} \times 100 \%
$$

where, $M_{\mathrm{NaOH}}=0.1 \mathrm{M}, m$ is the dry weight of the DANC sample in $g$, and 162 is the approximate molecular weight of the repeating unit in DANC. Each set of tests was conducted in triplicate. In addition to the DANC with $\mathrm{AC} 40 \%$, two other samples of DANC were prepared by varying the reaction time to $16 \mathrm{~h}$ and $18 \mathrm{~h}$, which resulted in AC of 24 and $32 \%$, respectively.

\subsection{Preparation of DCNC from DANC}

DANC with AC 24, 32, and 40\% were converted separately into DCNC by an oxidation reaction. It was considered that all the aldehyde groups in DANC are converted to carboxyl groups in DANC during the conversion process. In a typical process, $4.0 \mathrm{~g}$ of $\mathrm{NaCl}, 2.0 \mathrm{~g}$ of $\mathrm{NaClO}_{2}$ and $5.0 \mathrm{~mL}$ of $\mathrm{H}_{2} \mathrm{O}_{2}$ were added to $150 \mathrm{~mL}$ aqueous suspension $\left(0.001223 \mathrm{~g} \mathrm{~L}^{-1}\right)$ of DANC in a round bottom flask. The reaction mixture was stirred (400 rpm) for $24 \mathrm{~h}$ at room temperature. During this process, the reaction mixture turned into a yellow color solution which confirmed the oxidation of aldehyde groups to carboxyl groups. An excess amount of ethanol was slowly added into the reaction mixture which resulted in a gelatinous precipitate of DCNC. The precipitate was washed with DI water and the resultant suspension was then stored in a refrigerator at about $4-10{ }^{\circ} \mathrm{C}$. The prepared DCNCs with carboxylate content 24,32 , and $40 \%$ were marked as DCNC-24, DCNC-32, and DCNC-40, respectively.

\subsection{Preparation of PAA nanocomposite hydrogel}

PAA nanocomposite hydrogels were prepared by conducting free radical polymerization reactions with a fixed amount of monomer, crosslinker, and initiator in the presence of various amounts of functionalized NCC filler materials. For the preparation of PAA-DCNC-40 nanocomposite hydrogel, a total of $5.5 \mathrm{~mL}$ precursor solution was prepared with $0.03 \mathrm{~mol}$ of acrylic acid monomer, 0.1 mol\% MBA crosslinker (with respect to monomer), $0.1 \mathrm{~mol} \%$ APS initiator (with respect to monomer) and $0.058 \mathrm{wt} \%$ (with respect to monomer) of DCNC-40. The calculated concentration of acrylic acid was $5.2 \mathrm{M} . \mathrm{N}_{2}$ gas was purged into the solution for $4 \mathrm{~h}$. The solution was then poured into a rectangular cell consisting of two parallel glass plates separated by a Teflon spacer. The polymerization reaction was conducted at $65{ }^{\circ} \mathrm{C}$ temperature for $24 \mathrm{~h}$ in a controlled temperature oven. PAA-DCNC-24 and PAA-DCNC-32 nanocomposite hydrogels were prepared following the same procedure by adding $0.058 \mathrm{wt} \%$ (with respect to the monomer) of DCNC-24 and DCNC-32, respectively. For the preparation of PAA-NCC and PAA-DANC-40 hydrogels, $0.058 \mathrm{wt} \%$ of NCC and
DANC (AC-40\%) were added separately into the precursor solutions. Neat PAA hydrogel was prepared in the absence of any filler material. The concentration of acrylic acid was maintained at 5.2 $\mathrm{M}$ for all hydrogels.

\subsection{Characterization}

Freeze-dried samples of NCC, DANC, DCNC and PAA-DCNC were used for Fourier transform infrared spectroscopic (FTIR) analysis. A small amount of prepared material was mixed with $\mathrm{KBr}$ powder for making pellets. FTIR spectra were recorded in the range of $4000-400 \mathrm{~cm}^{-1}$ in transmission mode using an FTIR-8400 instrument (Shimadzu, Japan). Morphological images of the prepared materials were recorded using a field emission scanning electron microscope (FESEM), JSM-7600F (JEOL, Japan) at a $10.0 \mathrm{kV}$ operating voltage. A drop of dilute aqueous suspension of the samples was taken in a glass plate and vacuum dried, and the surface was coated with a thin layer of gold. X-ray diffraction (XRD) patterns of the freeze-dried samples were recorded using an X-ray diffractometer (RIGAKU Ultima IV, X-ray Diffractometer, Japan), with a $\mathrm{Cu}$ X-ray source (wavelength: $K \alpha_{1}=1.540598 \AA$ and $K \alpha_{2}=1.544426 \AA$ ) in the 10 to $50^{\circ} 2 \theta$ range. The crystallinity index was determined using the following equation from an experimental diffractogram, ${ }^{44}$

$$
\text { Crystallinity index }=\frac{I_{200}-I_{\mathrm{AM}}}{I_{200}} \times 100 \%
$$

Here, the intensity of the (200) planes is denoted by $I_{200}$, which is typically located around $2 \theta=22.6^{\circ} ; I_{\mathrm{AM}}$ is the lowest intensity between the planes (110) and (200). $I_{\mathrm{AM}}$ is the intensity at $2 \theta=18^{\circ}$, corresponding to the minimum in a diffractogram.

\subsection{Mechanical tests}

The tensile measurements were conducted using the universal testing machine UTM 100-P-250-12 (TestResources, USA). For tensile tests, dumbbell-shaped samples were cut; the length, width, and thickness were 10, 6, and $1 \mathrm{~mm}$, respectively. The elongation speed of the tensile test was $100 \mathrm{~mm} \mathrm{~min}^{-1}$. Stress was calculated following the equation $\sigma=F / A$, where $F$ is the recorded load, and $A$ is the cross-section area of the specimen, and the strain $(\varepsilon)$ of the samples was calculated from the ratio of elongation of the sample length $(\Delta l)$ compared to the initial length $\left(l_{\mathrm{o}}\right), \varepsilon=\Delta l / l_{\mathrm{o}}{ }^{12}$ The Young's modulus was calculated from the initial slope of the stress-strain curve. The toughness of each specimen was calculated by integrating the area underneath the stress-strain profile obtained from the tensile test.

\subsection{Swelling ratio measurements}

Small pieces of the as-prepared hydrogel samples of approximately similar weight were immersed in several beakers containing $500 \mathrm{~mL}$ of buffer solutions of different $\mathrm{pH}$ (3, 7 and 10). The buffer solutions were prepared by the following methods reported by Jabbari et $a l .{ }^{45}$ For the preparation of the buffer solution of $\mathrm{pH} 3,100 \mathrm{~mL}$ of $0.1 \mathrm{M}$ potassium hydrogen phthalate was mixed with $44.6 \mathrm{~mL}$ of $0.1 \mathrm{M} \mathrm{HCl}$, and the final volume was adjusted to $500 \mathrm{~mL}$. To prepare $500 \mathrm{~mL}$ of $\mathrm{pH} 7$ buffer, $100 \mathrm{~mL}$ of $\mathrm{KH}_{2} \mathrm{PO}_{4}$ was mixed with $58.2 \mathrm{~mL}$ of $0.1 \mathrm{M} \mathrm{NaOH}$ and water. 
The basic buffer of $\mathrm{pH} 10$ was prepared by mixing $100 \mathrm{~mL}$ of $0.05 \mathrm{M} \mathrm{NaHCO}_{3}$ with $21.4 \mathrm{~mL}$ of $0.1 \mathrm{M} \mathrm{NaOH}$ and the final volume was adjusted to $500 \mathrm{~mL}$. The samples were swelled at room temperature. After the swelling for the desired time, the swelled gels were removed from the water and weighed. The weight of the samples was recorded at different time intervals of $24,48,72,96,120$, and $144 \mathrm{~h}$. The swelling ratios of the samples were calculated using the following equation:

$$
\mathrm{SR}=\frac{W_{\mathrm{t}}-W_{\mathrm{d}}}{W_{\mathrm{d}}}
$$

where, $W_{\mathrm{t}}$ and $W_{\mathrm{d}}$ were the weights of the swollen and dry hydrogel, respectively.

\section{Results and discussion}

NCC, DANC, and DCNC were prepared from MCC applying an acidic hydrolysis process. Different factors affecting the acidic hydrolysis of cellulose were examined in earlier reported works. The standard parameters were reaction time and temperature, type of acid, acid concentration, the ratio of MCC to acid, and the effect of applied external energy. ${ }^{46-48}$ In the present study, the chemical degradation mechanism is the acid-catalyzed cleavage of the $\beta-1-4$-glycosidic bond. A schematic diagram of the preparation of NCC, DANC, and DCNC has been presented in Fig. 1. Sulfuric acid is beneficial for the hydrolysis of NCC, because of its accessibility to the amorphous domains. The amorphous areas have lower density due to the random orientation of the polymers in these regions. It is more exposed to acid and especially to the penetration of hydronium ions $\mathrm{H}_{3} \mathrm{O}^{+}$leading to hydrolytic cleavage of the $\beta$-1-4-glycosidic bond. ${ }^{49}$ The application of ultrasonic energy after hydrolysis has been applied to reduce the agglomeration. Sulfuric acid also helps to reduce the agglomeration by introducing negative charges on the NCC surface. Sodium meta-periodate is a kind of selective oxidizing reagent which selectively cleaves the bond between $\mathrm{C}-2$ and $\mathrm{C}-3$, and converts the vicinal hydroxyl groups to aldehyde. ${ }^{50,51}$ The reaction is susceptible to light, temperature and reaction time; as a result, they are carefully selected in our preparation conditions. After the desired extent of oxidation, the reaction was quenched by ethylene glycol. ${ }^{52}$ The AC of the DANC was determined by the oxime formation method, as stated in the Experimental section. Periodate oxidation is known to be a highly specific reaction to convert 1,2-dihydroxy groups (glycol) to dialdehyde groups without significant side reactions. ${ }^{50}$ During the oxidation, $1 \mathrm{~mol}$ of glucose unit of NCC theoretically consumes $1 \mathrm{~mol}$ of $\mathrm{NaIO}_{4} \cdot{ }^{53,54}$ The degree of oxidation, which corresponds with the AC is generally determined by measuring the number of reactive aldehydes available for the oxime formation. Since cellulose is a polymer, only surface hydroxyl groups will react with the oxidant, and 100\% conversion is not expected. In this study, it was found that about $40 \%$ of vicinal hydroxyl groups were converted to a dialdehyde group. The prepared aldehyde groups of DANC, in turn, exhibited high reactivity toward further modification, and they were selectively oxidized to acids to form chemically stable sodium salts of 2,3-dicarboxylic acid nanocrystalline cellulose by the reaction with sodium chlorite, sodium chloride, and hydrogen peroxide. ${ }^{42}$ During the conversion of aldehyde to acid, it was considered that the amount of acid content is equal to that of aldehyde as no side reactions are expected.

To confirm the preparation of DANC and DCNC from NCC, the FTIR spectra of the materials were recorded using a spectrophotometer and compared in Fig. 2. In NCC, DANC, and DCNC the broad peaks at $3400 \mathrm{~cm}^{-1}$ were attributed to the $\mathrm{O}-\mathrm{H}$ stretching bands, and the peaks at $2900 \mathrm{~cm}^{-1}$ corresponded to the presence of aliphatic saturated symmetric $\mathrm{C}-\mathrm{H}$ stretching vibrations of cellulose. ${ }^{55}$ The absorption peaks observed at $\sim 1640 \mathrm{~cm}^{-1}$ and $1370 \mathrm{~cm}^{-1}$ were caused by the $\mathrm{O}-\mathrm{H}$ bending modes of absorbed water and $\mathrm{C}-\mathrm{H}$ bending within the polysaccharide rings of cellulose, respectively. ${ }^{56}$ FTIR peaks at $1058 \mathrm{~cm}^{-1}$ corresponded to the $\mathrm{C}-\mathrm{O}-\mathrm{C}$ pyranose ring stretching vibration and that at $898 \mathrm{~cm}^{-1}$ was due to $\beta$-glycosidic linkages of cellulose. ${ }^{57,58}$ When FTIR spectra of DANC and DCNC are compared with the NCC, it is evident that the typical structure of the parent cellulose backbone was preserved after the oxidation process. The characteristic weak peak for the free aldehyde was observed at $1740 \mathrm{~cm}^{-1}$ in the case of DANC only. Such peaks are challenging to identify due to the moisture content. Spedding et al. ${ }^{59}$ concluded from his findings that on drying, DANC-type oxidized samples have several forms of combined aldehyde grouping, viz, hydrated aldehyde, hemiacetal, etc.
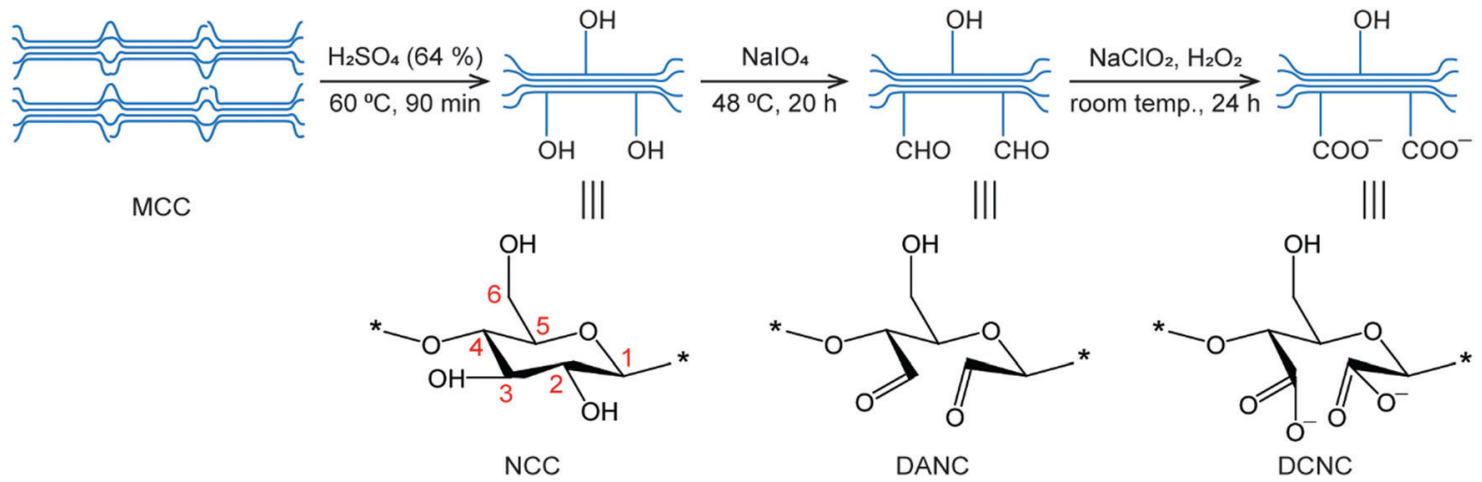

Fig. 1 Schematic diagram of the preparation of DANC and DCNC from MCC via NCC. 


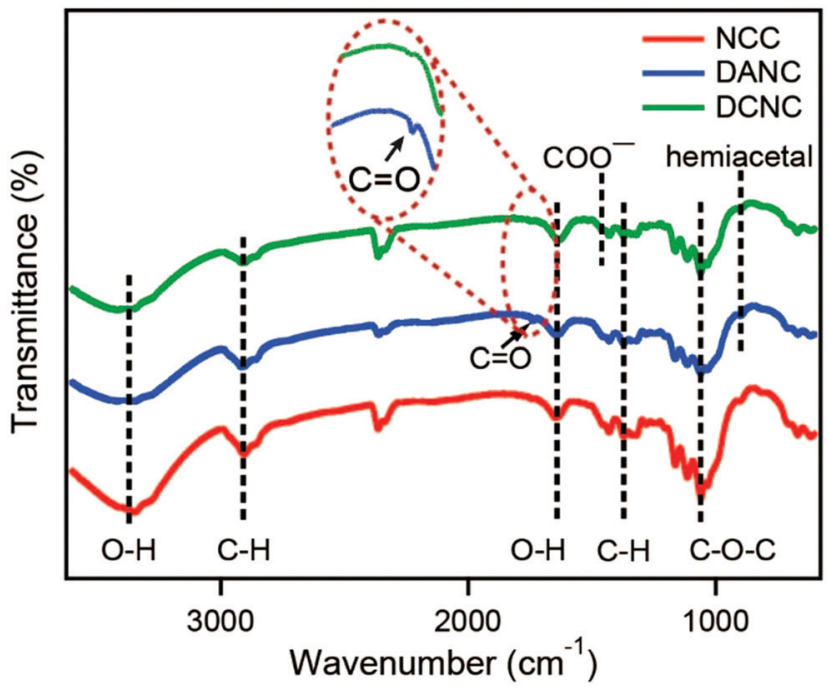

Fig. 2 Comparison of the FTIR spectra of NCC (red), DANC-40 (blue) and DCNC-40 (green).

They also confirmed the presence of hemiacetal by the peak at around $900 \mathrm{~cm}^{-1}$. Here, absorption at about $900 \mathrm{~cm}^{-1}$ is present in the case of NCC and DANC. However, such a peak is almost absent in DCNC. Some authors claimed that such an absorption peak might appear at around $890 \mathrm{~cm}^{-1}$ and observed that the peak intensity could increase due to oxidation. ${ }^{60}$ However, the absorption peak at $898 \mathrm{~cm}^{-1}$ was also reported for rocking vibrations of $\mathrm{CH}_{2}$ in the cellulose. ${ }^{61}$ As a result, it is challenging to distinguish NCC and DANC by this peak. However, such a peak completely disappears in the case of DCNC due to the conversion of aldehyde to acid. In our work, the carboxylate functionalization in DCNC by the chlorite-oxidation is thus evident from the disappearance of the aldehyde $\mathrm{C}=\mathrm{O}$ band at $1740 \mathrm{~cm}^{-1}$ and the reduction of the intensity of the hemiacetal peak at $900 \mathrm{~cm}^{-1}$.

In addition to FTIR, the wet chemical method was applied to confirm the corresponding conversions. The introduction of aldehyde groups in NCC and corresponding conversion to acids was confirmed chemically by 2,4-DNPH. The yellow-colored solution of 2,4-DNPH was added separately to test tubes containing a dilute aqueous suspension of NCC, DANC-40, and a solution of DCNC-40. The color change of the solutions was observed. The images of the test tubes after adding 2,4-DNPH are demonstrated in Fig. 3. The test tube containing DANC-40 formed an orange precipitate due to the characteristic reaction of the aldehyde with the reagent resulting in hydrazone product. The precipitate formation was not observed in the test tube containing NCC. NCC transformed into a turbid greenishyellow solution due to its aqueous insolubility. A transparent yellow solution was found with the addition of 2,4-DNPH in the solution of DCNC-40, indicating no chemical reactions with carboxylic groups. The result suggests the incorporation of aldehyde groups in NCC and the complete conversion of aldehyde groups into carboxylic ones.

The surface morphologies of NCC and functionalized NCCs were investigated by FESEM. The FESEM images of NCC,

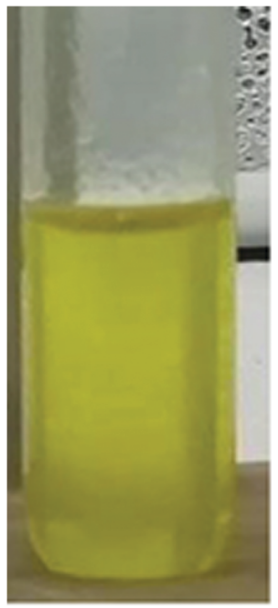

NCC

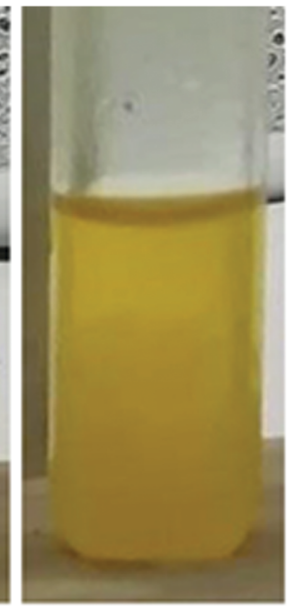

DANC

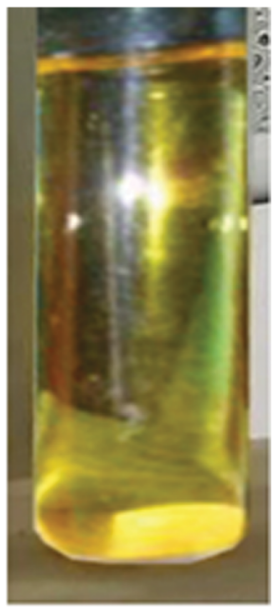

DCNC
Fig. 3 Images of test tubes after conducting 2,4-DNPH wet chemical tests with NCC (greenish yellow), DANC-40 (orange ppt) and DCNC-40 (transparent yellow).

DANC-40, and DCNC-40 are presented in Fig. 4(a), (c) and (d), respectively. Changes in surface morphologies for the functionalization of NCC were observed from these images. Compact agglomerated needle-shaped cellulose nanocrystals with an average length of $120.5 \mathrm{~nm}$ were observed from the FESEM micrograph of NCC (Fig. 4(a)). ${ }^{62,63}$ The diameter of the as-prepared NCC was measured by Image J software and demonstrated a wide range of distribution (Fig. 4(b)). The average diameter was $19.5 \mathrm{~nm}$ with a standard deviation of $0.3 \mathrm{~nm}^{62}$ The compactness of the NCCs suggested that cellulosic chains had strong intermolecular hydrogen bondings. However, from the images of DANC-40 (Fig. 4(c)) and DCNC-40 (Fig. 4(d)) it is clear that after periodate oxidation, the fiber diameter was reduced. The disintegration of cellulose fibers by the periodate oxidation is responsible for this reduction in size. ${ }^{64}$ Large agglomeration in DCNC might be due to the high degree of hydrophilic nature of the material.

$\mathrm{XRD}$ analysis was performed to investigate the changes in the crystalline behavior of the prepared materials during the conversion of NCC to DANC and DCNC. The XRD profiles of NCC, DANC-40 and DCNC-40 are compared in Fig. 5. All the samples exhibited similar peaks at around the angles $2 \theta=14.8^{\circ}, 16.7^{\circ}, 22.6^{\circ}$, and $34.6^{\circ}$ corresponding to the (1Mx0031;0), (110), (200), and (004) planes, which are reasonable for typical cellulose structures. ${ }^{58,65,66}$ It is evident that no extensive change in crystallinity occurred during the conversion. However, the small variation in the peak intensities represented a change in polymeric alignment in NCC during the modification, which is consistent with the previously reported results. ${ }^{67}$ The crystallinity index (C.I.) of the samples was calculated according to the amorphous subtraction method. The C.I. of the starting NCC was $91.24 \%$, which decreased to $88.14 \%$ when converted to DANC and further decreased to $75.00 \%$ in DCNC. These results are considerably consistent with the observation reported by Kim $e t$ al., where 

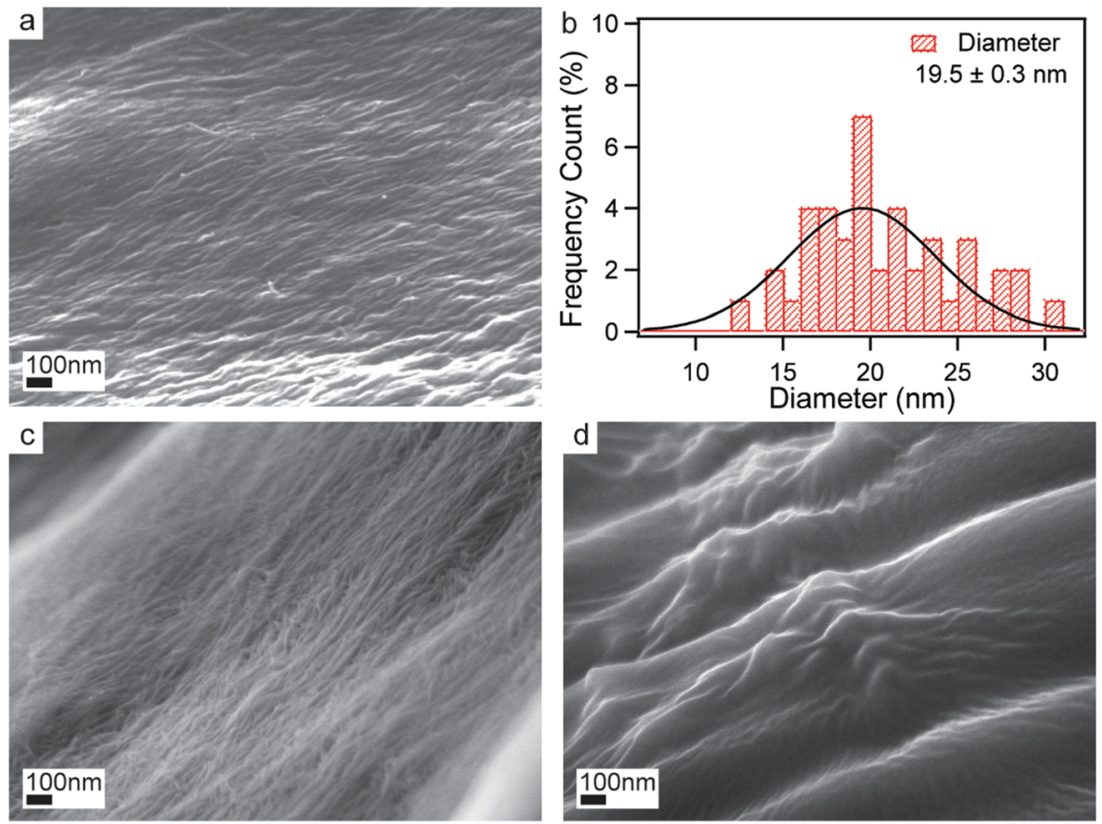

Fig. 4 FESEM images of NCC (a), DANC-40 (c) and DCNC-40 (d). Particle size distribution (diameter) of NCC (b).

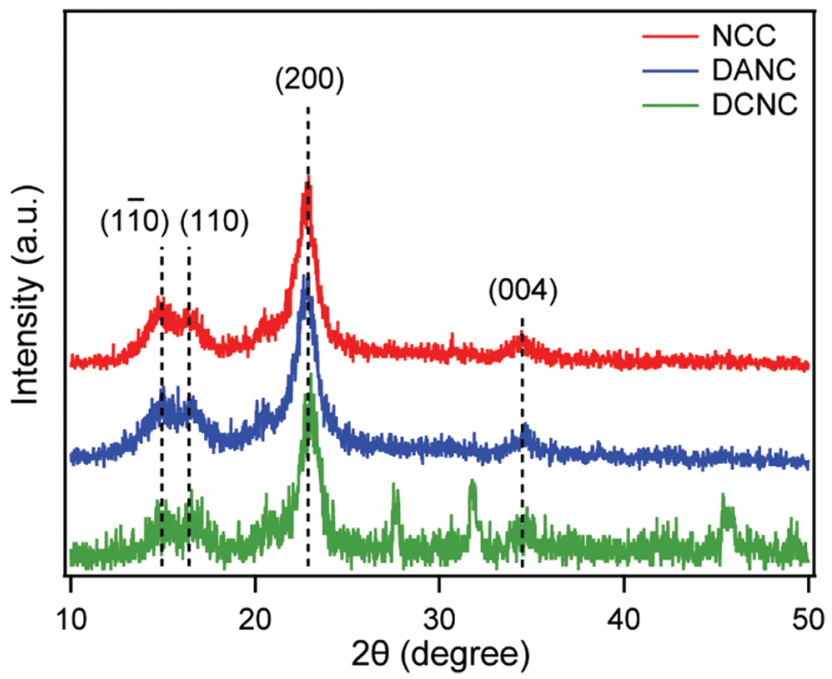

Fig. 5 Comparison of the XRD patterns of NCC, DANC-40 and DCNC-40.

the C.I. was found to be reduced with the increased level of oxidation by periodate and chlorite. ${ }^{53}$ Here, the loss of crystallinity is attributed to the opening of glucopyranose rings and destruction of the ordered packing present in the cellulose materials. ${ }^{68}$ Additionally, the decrease in C.I. might have also resulted from the degradation and changes in the hydrogen bonding in the materials. ${ }^{69}$

To investigate the influence of the NCC and functionally modified NCC on the material properties of PAA nanocomposite hydrogels, PAA hydrogels were prepared by a free radical polymerization reaction of AA monomer in the presence of NCC, DANC, and DCNC separately. To initiate the reaction, APS was used as an initiator, and a small amount of conventional covalent crosslinker MBA was added to maintain the relative structural integrity of the prepared hydrogels. A scheme of the preparation process has been demonstrated in Fig. 6(a). Here, PAA polymer chains are covalently crosslinked by MBA. Additionally, the surface functional groups of the NCC, DANC, and DCNC are expected to be responsible for additional physical crosslinking. Eventually, both the covalent crosslinker and physical crosslinker are expected to influence the mechanical behavior of the matrix of the hydrogels.

The FTIR spectrum of the DCNC-40 incorporated PAA hydrogel is shown in Fig. 6(b). The absorption peaks related to the polysaccharide rings of cellulose were observed in the spectrum. The peaks at $1640 \mathrm{~cm}^{-1}$ and $1375 \mathrm{~cm}^{-1}$ correspond to the $\mathrm{O}-\mathrm{H}$ and $\mathrm{C}-\mathrm{H}$ bending modes, respectively, present in the glucose moieties of DCNC. Also, the peaks associated with $\mathrm{C}-\mathrm{O}-\mathrm{C}$ pyranose ring stretching vibration $\left(1058 \mathrm{~cm}^{-1}\right)$ and $\beta$-glycosidic linkages $\left(898 \mathrm{~cm}^{-1}\right)$ were observed. The bands at 1409 and $1450 \mathrm{~cm}^{-1}$ in the spectrum were attributed to $\mathrm{C}-\mathrm{OH}$ bending vibrations related to acrylic acid. ${ }^{31}$ These observations suggested that DCNC was successfully incorporated into the matrix of the PAA hydrogel. The characteristic peak at $1550 \mathrm{~cm}^{-1}$ was observed corresponding to the $\mathrm{C}-\mathrm{N}$ stretching vibration. ${ }^{70}$ A vibration band between $1300-1200 \mathrm{~cm}^{-1}$ was evident due to the in-phase combination of the $\mathrm{N}-\mathrm{H}$ bending and the $\mathrm{C}-\mathrm{N}$ stretching vibrations. ${ }^{70}$ They indicate the presence of MBA covalent crosslinker in the PAA-DCNC-40 hydrogel. The XRD analysis of the PAA-DCNC-40 nanocomposite hydrogel was conducted, and the XRD spectrum has been shown in Fig. 6(c). The PAA-DCNC-40 hydrogel seems to be amorphous. A broad band was found between the $2 \theta$ of 5 to $80^{\circ}$. The broad spectrum around $20^{\circ}$ is the characteristic amorphous peak of PAA. However, a peak at $22.7^{\circ}$ indicated the presence of some semicrystalline material in the PAA-DCNC-40 hydrogel. 

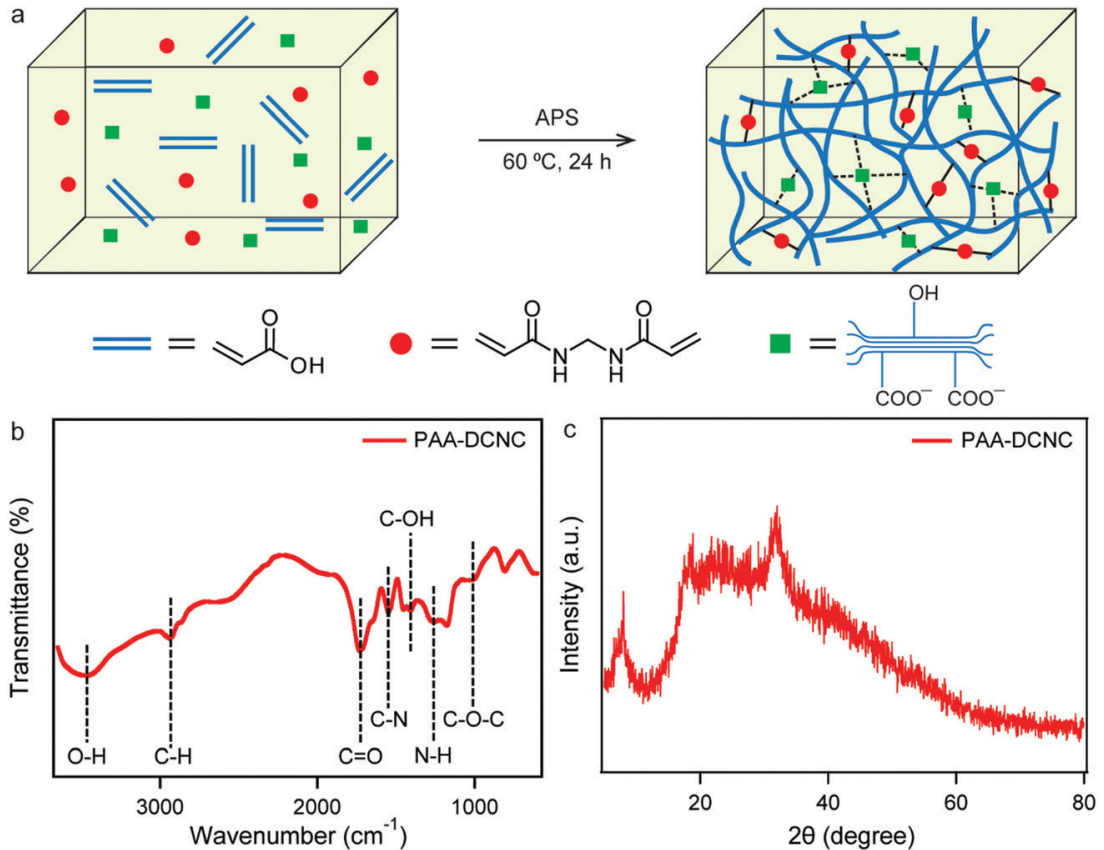

Fig. 6 Schematic illustration of the preparation of DCNC-PAA nanocomposite hydrogel (a), and FTIR (b) and XRD (c) spectrum of the prepared PAA-DCNC-40 hydrogel.

Tensile tests of the prepared hydrogels were conducted to investigate the mechanical properties. The comparative stressstrain profiles of the PAA, PAA-NCC, PAA-DANC-40, and PAADCNC-40 hydrogels are demonstrated in Fig. 7(a). For this comparative study, an optimized fixed amount of $0.058 \mathrm{wt} \%$ of filler materials was incorporated into these hydrogels. For a higher percentage of NCC and DANC incorporation, the homogeneity of the hydrogels was lost. The formed gels were brittle and weak. This might be due to the agglomeration behavior and poor solubility of NCC and DANC in water. The parameters related to mechanical properties of the hydrogels determined from the figure are presented in Table 1. The addition of nanocrystalline cellulosic materials, irrespective of their type of modifications, improved the mechanical properties of neat PAA. The Young's modulus, tensile strength, and toughness of the weak PAA hydrogel were increased. Here, the PAA-DCNC-40 nanocomposite hydrogel demonstrated the best mechanical performance. The Young's modulus of neat PAA hydrogel was only $90.2 \mathrm{kPa}$, which was increased by about six times (619.1 kPa) by the addition of a minimal amount (0.058 wt\%) of DCNC with $40 \%$ carboxylation. Six times enhancement in the tensile strength was observed in the presence of DCNC-40. The toughness of the PAA-DCNC-40 hydrogel was $522.2 \mathrm{~kJ} \mathrm{~m}^{-3}$, which was only $97.4 \mathrm{~kJ} \mathrm{~m}^{-3}$ in the absence of DCNC.

The improvement in mechanical behavior was attributed to the large number of physical crosslinking bonds formed by DCNC in the PAA hydrogel matrix. The polar functional carboxyl groups of DCNC strengthen the physical interactions and generate crosslinking in the network resulting in a higher stiffness of the PAADCNC hydrogels. The dicarboxylate modified NCC with a large number of carboxyl groups was expected to strengthen the physical interaction. This type of improvement is a similar strategy as reported for other nanocomposites. ${ }^{71}$ But our proposed approach to improve the mechanical properties of the hydrogel by the incorporation of functionalized NCC is beneficial because of the biodegradable nature of the selected nanomaterial. The mechanical
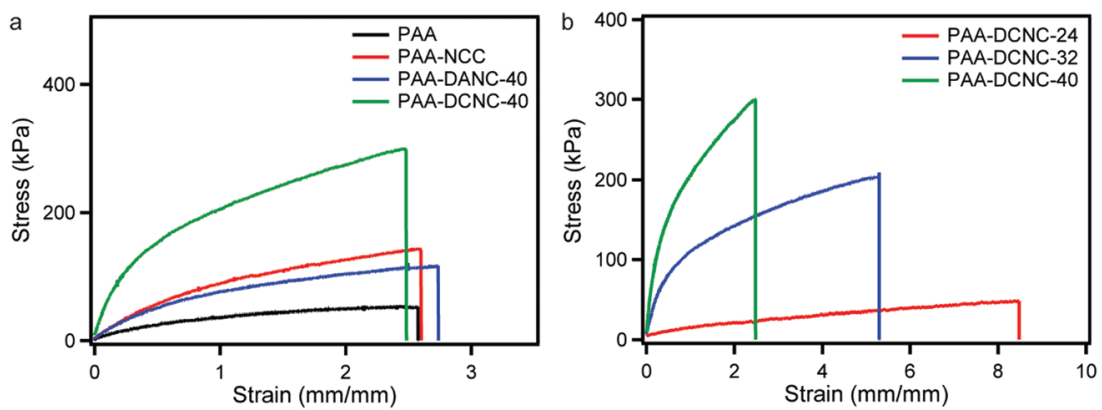

Fig. 7 Stress-strain curves of PAA composite hydrogels prepared with different cross-linkers (a) and PAA-DCNC hydrogels with different compositions of DCNC (b). 
Table 1 Mechanical parameters calculated from the stress-strain profiles of the prepared hydrogels. The PAA-NCC, PAA-DANC-40, PAA-DCNC-24, PAA-DCNC-32 and PAA-DCNC-40 hydrogels were prepared with a fixed amount, 0.058 wt\% of NCC, DANC-40, DCNC-24, DCNC-32 and DCNC-40, respectively

\begin{tabular}{lccr}
\hline Hydrogels & \multicolumn{1}{l}{$\begin{array}{l}\text { Young's } \\
\text { modulus }(\mathrm{kPa})\end{array}$} & $\begin{array}{l}\text { Tensile } \\
\text { strength }(\mathrm{kPa})\end{array}$ & \multicolumn{1}{c}{$\begin{array}{l}\text { Toughness } \\
\left(\mathrm{kJ} \mathrm{m}^{-3}\right)\end{array}$} \\
\hline PAA & $90.2 \pm 2.1$ & $53.2 \pm 1.3$ & $97.5 \pm 2.3$ \\
PAA-NCC & $170.0 \pm 2.9$ & $144.5 \pm 2.7$ & $243.8 \pm 4.4$ \\
PAA-DANC-40 & $170.0 \pm 2.5$ & $124.3 \pm 1.9$ & $219.6 \pm 2.9$ \\
PAA-DCNC-24 & $67.6 \pm 2.1$ & $48.1 \pm 1.3$ & $259.2 \pm 8.2$ \\
PAA-DCNC-32 & $297.5 \pm 3.9$ & $209.2 \pm 2.9$ & $786.3 \pm 10.5$ \\
PAA-DCNC-40 & $619.1 \pm 6.2$ & $300.9 \pm 3.4$ & $522.2 \pm 5.7$
\end{tabular}

properties of the PAA hydrogel were also improved by the addition of NCC; however, not to the extent where DCNC improved.

The polar hydroxyl groups are responsible for making physical bonding in the polymer matrix. The addition of DANC-40 to PAA moderately improved the mechanical performance. The tensile strength of the PAA-NCC hydrogel is higher than that of the PAA-DANC-40 hydrogel. From these observations, it is clear that DCNC is strongly capable of forming physical bonding in the PAA matrix. In contrast, NCC is moderate and DANC is weakly capable of making intermolecular physical bonding. The stiffness of the PAA matrix is significantly influenced by the extent of the intermolecular bonding. It was also observed that the mechanical performance of the PAA-DCNC nanocomposite hydrogel is relatively dependent on the carboxylate content of DCNC (Fig. 7(b)). The PAA hydrogels with a low degree of carboxylate modification demonstrated small values of tensile strength and modulus. It indicates the influential role of additional intra- and inter-molecular hydrogen bonds arising from the carboxylate groups for the improved mechanical properties. The carboxyl groups and crystallinity also have an impact on the mechanical properties. The highest carboxylate content DCNC resulted in the most significant values of mechanical parameters. The nanocrystalline materials consist of molecular chains that are tightly packed in an ordered and parallel arrangement. They are highly capable of dissipating high energy in a polymer matrix if the deformation force is applied. This type of energy dissipation to enhance the toughness is possible for NCC, DANC, and DCNC. However, here, the PAA matrix is greatly influenced by the DCNC-40 compared to DANC-40 and NCC because of the great ability to form reliable physical bonding sites by mainly polar carboxylic groups. It is worth mentioning that the large extent of the hydrophilic nature of DCNC which caused the agglomeration of the material, might have positively contributed to the attractive interaction with the polar matrix of PAA and strengthened the hydrogel. It has been observed that the mechanical properties of PAA hydrogel have been significantly improved by the incorporation of carboxylate modified NCC. This kind of process will be helpful in finding alternatives to synthetic crosslinkers like MBA, which frequently incur non-biodegradable and non-biocompatible properties in hydrogels.

The swelling behavior of the PAA, PAA-NCC, PAA-DANC-40, and PAA-DCNC-40 hydrogels was observed as a function of time

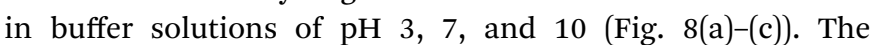
prepared hydrogels in all buffer solutions demonstrated an initial sharp rise in swelling with time, which reached a fixed equilibrium swelling for a long time. All of the prepared PAA hydrogels demonstrated the highest swelling capacity at $\mathrm{pH} 7$ compared to that at $\mathrm{pH} 3$ and 10. This variation of swelling behavior with $\mathrm{pH}$ is common for nanocomposite hydrogels with a PAA matrix and consistent with the results reported by Sujan et al. for nanoslilica-PAA hydrogels. ${ }^{9}$ At $\mathrm{pH} 7$, a huge osmotic pressure is generated in the PAA-composite matrix by the formation of a large number of carboxylate groups $\left(\mathrm{COO}^{-}\right)$. Among the three types of nanocomposite hydrogels, the swelling capacity of PAA-DCNC-40 is significantly high at $\mathrm{pH} 7$ due to the presence of additional carboxylate groups $\left(\mathrm{COO}^{-}\right)$in the matrix (Fig. 8(b)). In addition to that, the high extent of conformational changes in the polymeric backbone of DCNC caused by the electrostatic repulsions of the carboxylate groups during the swelling contributed to this high-water uptake. However, at $\mathrm{pH} 3$, most of the carboxylic groups of the PAA matrix remain protonated $\left(\mathrm{COO}^{-} \rightarrow \mathrm{COOH}\right)$. This causes the formation of intra- and intermolecular hydrogen bonding and some additional physical crosslinking, thus reducing the swelling capacity of the PAA-NCC and PAA-DCNC-40 hydrogels. ${ }^{72,73}$ These effects were not prominent in the PAA-DANC-40 hydrogel due to the presence of $-\mathrm{CHO}$ groups and demonstrated the
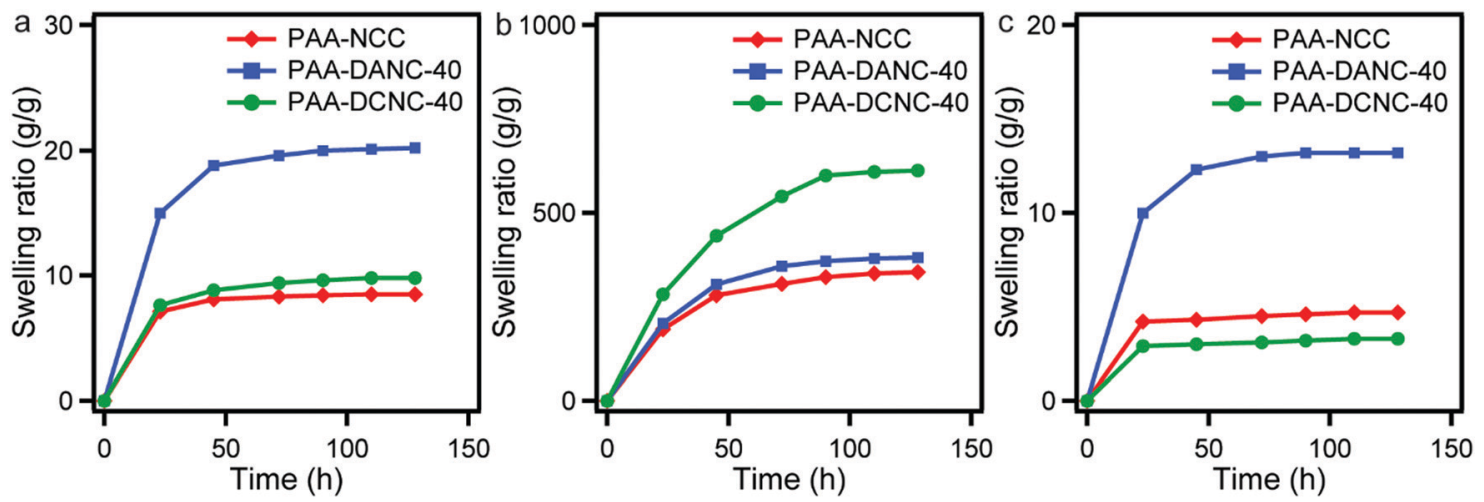

Fig. 8 Time dependent swelling ratio of the PAA nanocomposite hydrogels at $\mathrm{pH}=3$ (a), $\mathrm{pH}=7$ (b), and $\mathrm{pH}=10$ (c). 
highest swelling ratio compared to PAA-NCC and PAA-DCNC-40 at $\mathrm{pH} 3$ (Fig. 8(a)). At $\mathrm{pH}$ 10, in highly basic solutions, the charge screening effect of the counter ions and high ionic strength of the swelling medium might have decreased the osmotic pressure in the polymer matrix of the PAA-NCC and PAA-DCNC-40 hydrogels (Fig. 8(c)). ${ }^{72,74}$ Here also, such effects are less prominent in the PAA-DANC-40 hydrogel containing - $\mathrm{CHO}$ groups and resulted in the highest swelling capacity compared to the PAA-NCC and PAA-DCNC-40 hydrogels.

It is evident that the PAA-DCNC hydrogels with improved mechanical properties have demonstrated $\mathrm{pH}$ sensitive swelling behavior. These kinds of hydrogels have promising application in biomedical engineering. K. Haraguchi et al. pointed out that due to the high-water content and cross-linking structure, some commonly used hydrogels such as polyethylene glycol (PEG) and agarose usually yield very low stretchability and compressibility, which is a major drawback for the application of the material in soft robotics. ${ }^{75}$ Here, the PAA-DCNC hydrogels have exhibited enhanced stimuli responsiveness with improved mechanical performance compared to neat PAA hydrogels, which could further explore the application of the hydrogel in future biomimetic machines and soft robotics systems. ${ }^{76}$

\section{Conclusions}

In this research, NCC and its functionally modified products, DANC and DCNC, were successfully incorporated into the PAA matrix through a one-step polymerization technique. A remarkable enhancement in the mechanical strength, modulus, and toughness was observed in the prepared PAA-NCC, PAA-DANC, and PAA-DCNC nanocomposite hydrogels compared to the neat PAA hydrogel. The polarity of the functional groups attached to the nanomaterial greatly influenced the mechanical properties. The most considerable improvement was observed in the PAA-DCNC hydrogels. The excellent mechanical performance was attributed to the multifunctional bond forming ability of DCNC with the polymer matrix. Also, the swelling behaviors of the prepared hydrogels were affected by the presence of different functionalities of cellulosic nanomaterials. The swelling properties were highly pH-dependent, and the highest swelling was observed at $6<$ $\mathrm{pH}<10$ for PAA-DCNC. Finally, a systematic approach for the simple modification of biodegradable NCC and their influential role for the improvement of different properties of PAA hydrogels was presented, which will significantly impact the viable applications of this material in the area of bioengineering.

\section{Conflicts of interest}

There are no conflicts to declare.

\section{Acknowledgements}

The authors thankfully acknowledge the financial support from the Bangladesh University of Engineering and Technology
(BUET) and the Ministry of Science and Technology (Government of the People's Republic of Bangladesh) for this research.

\section{References}

1 X. Chen, M. Wang, X. Yang, Y. Wang, L. Yu, J. Sun and J. Ding, Theranostics, 2019, 9, 6080-6098.

2 Y. Zhuang, X. Yang, Y. Li, Y. Chen, X. Peng, L. Yu and J. Ding, ACS Appl. Mater. Interfaces, 2019, 11, 29604-29618.

3 X. Yang, X. Chen, Y. Wang, G. Xu and L. Yu, Chem. Eng. J., 2020, 396, 125320.

4 X. Chen, J. Zhang, K. Wu, X. Wu, J. Tang, S. Cui, D. Cao, R. Liu, C. Peng, L. Yu and J. Ding, Small Methods, 2020, 2000310.

5 R. Yoshida and Y. Uesusuki, Biomacromolecules, 2005, 6, 2923-2926.

6 S. Liang, L. Zhang and J. Xu, J. Membr. Sci., 2007, 287, 19-28.

7 N. Bhattarai, J. Gunn and M. Zhang, Adv. Drug Delivery Rev., 2010, 62, 83-99.

8 K. Haraguchi, T. Takehisa and S. Fan, Macromolecules, 2002, 35, 10162-10171.

9 M. I. Sujan, S. D. Sarkar, S. Sultana, L. Bushra, R. Tareq, C. K. Roy and M. S. Azam, RSC Adv., 2020, 10, 6213-6222.

10 Z. Liu, Z. Yang and Y. Luo, Polym. Compos., 2012, 33, 665-674.

11 M. K. Shin, G. M. Spinks, S. R. Shin, S. I. Kim and S. J. Kim, Adv. Mater., 2009, 21, 1712-1715.

12 S. D. Sarkar, M. M. Uddin, C. K. Roy, M. J. Hossen, M. I. Sujan and M. S. Azam, RSC Adv., 2020, 10, 10949-10958.

13 M. Patenaude and T. Hoare, ACS Macro Lett., 2012, 1, 409-413.

14 T. Hoare, E. Bellas, D. Zurakowski and D. Kohane, J. Biomed. Mater. Res., Part A, 2009, 92, 586-595.

15 T. Hoare, E. Bellas, D. Zurakowski and D. S. Kohane, J. Biomed. Mater. Res., Part A, 2010, 92, 586-595.

16 Y. Habibi, L. A. Lucia and O. J. Rojas, Chem. Rev., 2010, 110, 3479-3500.

17 S. J. Eichhorn, Soft Matter, 2011, 7, 303-315.

18 M. J. Hossen, S. D. Sarkar, M. M. Uddin, C. K. Roy and M. S. Azam, ChemSelect, 2020, 5, 8906-8914.

19 N. Johar, I. Ahmad and A. Dufresne, Ind. Crops Prod., 2012, 37, 93-99.

20 S. Thambiraj and D. R. Shankaran, Appl. Surf. Sci., 2017, 412, 405-416.

21 J. Araki, M. Wada, S. Kuga and T. Okano, Colloids Surf., A, 1998, 142, 75-82.

22 M. R. Sucaldito and D. H. Camacho, Carbohydr. Polym., 2017, 169, 315-323.

23 B. G. Rånby, Discuss. Faraday Soc., 1951, 11, 158-164.

24 A. C. Leung, S. Hrapovic, E. Lam, Y. Liu, K. B. Male, K. A. Mahmoud and J. H. Luong, Small, 2011, 7, 302-305.

25 D. Liu, T. Zhong, P. R. Chang, K. Li and Q. Wu, Bioresour. Technol., 2010, 101, 2529-2536.

26 Q. Xu, Y. Ji, Q. Sun, Y. Fu, Y. Xu and L. Jin, Nanomaterials, 2019, 9, 253. 
27 T. Huq, S. Salmieri, A. Khan, R. A. Khan, C. Le Tien, B. Riedl, C. Fraschini, J. Bouchard, J. Uribe-Calderon and M. R. Kamal, Carbohydr. Polym., 2012, 90, 1757-1763.

28 S. A. Paralikar, J. Simonsen and J. Lombardi, J. Membr. Sci., 2008, 320, 248-258.

29 C. Zhou, Q. Wu, Y. Yue and Q. Zhang, J. Colloid Interface Sci., 2011, 353, 116-123.

30 T. Jayaramudu, H.-U. Ko, H. C. Kim, J. W. Kim and J. Kim, Materials, 2019, 12, 2080.

31 L. S. Lim, I. Ahmad and M. Lazim, Sains Malays., 2015, 44, 779-785.

32 M. Shahzamani, S. Taheri, A. Roghanizad, N. Naseri and M. Dinari, Int. J. Biol. Macromol., 2020, 147, 187-193.

33 D. Cheng, Y. Wen, L. Wang, X. An, X. Zhu and Y. Ni, Carbohydr. Polym., 2015, 123, 157-163.

34 X. Yang and E. D. Cranston, Chem. Mater., 2014, 26, 6016-6025.

35 D. Yang, X. Peng, L. Zhong, X. Cao, W. Chen, S. Wang, C. Liu and R. Sun, RSC Adv., 2015, 5, 13878-13885.

36 J. Yang, C.-R. Han, J.-F. Duan, M.-G. Ma, X.-M. Zhang, F. Xu and R.-C. Sun, Cellulose, 2013, 20, 227-237.

37 B. Dhuiège, G. Pecastaings and G. Sèbe, ACS Sustainable Chem. Eng., 2018, 7, 187-196.

38 J. Tang, J. Sisler, N. Grishkewich and K. C. Tam, J. Colloid Interface Sci., 2017, 494, 397-409.

39 L. Thompson, J. Azadmanjiri, M. Nikzad, I. Sbarski, J. Wang and A. Yu, Rev. Adv. Mater. Sci., 2019, 58, 1-16.

40 J. Yang, C.-R. Han, X.-M. Zhang, F. Xu and R.-C. Sun, Macromolecules, 2014, 47, 4077-4086.

41 T. Saito and A. Isogai, Biomacromolecules, 2004, 5, 1983-1989.

42 H. Liimatainen, M. Visanko, J. A. Sirviö, O. E. Hormi and J. Niinimaki, Biomacromolecules, 2012, 13, 1592-1597.

43 S. Veelaert, D. De Wit, K. Gotlieb and R. Verhé, Carbohydr. Polym., 1997, 33, 153-162.

44 L. Segal, J. Creely, A. Martin Jr and C. Conrad, Text. Res. J., 1959, 29, 786-794.

45 E. Jabbari and S. Nozari, Eur. Polym. J., 2000, 36, 2685-2692.

46 S. Beck-Candanedo, M. Roman and D. G. Gray, Biomacromolecules, 2005, 6, 1048-1054.

47 J. Araki, M. Wada, S. Kuga and T. Okano, J. Wood Sci., 1999, 45, 258-261.

48 E. L. Jackson and C. Hudson, J. Am. Chem. Soc., 1938, 60, 989-991.

49 M. Lima and R. Borsali, Macromol. Rapid Commun., 2004, 25, 771-787.

50 Y. Fang, R. Takahashi and K. Nishinari, Biomacromolecules, 2005, 6, 3202-3208.

51 B. T. Hofreiter, I. A. Wolff and C. L. Mehltretter, J. Am. Chem. Soc., 1957, 79, 6457-6460.
52 Y.-P. Wu, L. G. Miller and N. D. Danielson, Analyst, 1985, 110, 1073-1076.

53 U.-J. Kim, S. Kuga, M. Wada, T. Okano and T. Kondo, Biomacromolecules, 2000, 1, 488-492.

54 P. Calvini and A. Gorassini, Cellulose, 2012, 19, 1107-1114.

55 S. B. A. Hamid, S. K. Zain, R. Das and G. Centi, Carbohydr. Polym., 2016, 138, 349-355.

56 X. Y. Tan, S. B. A. Hamid and C. W. Lai, Biomass Bioenergy, 2015, 81, 584-591.

57 M. B. Yahya, H. V. Lee and S. B. Abd Hamid, BioResources, 2015, 10, 7627-7639.

58 A. Kumar, Y. S. Negi, V. Choudhary and N. K. Bhardwaj, J. Mater. Phys. Chem., 2014, 2, 1-8.

59 H. Spedding, J. Chem. Soc., 1960, 3147-3152.

60 J. Y. Kim and H.-M. Choi, Cellul. Chem. Technol., 2014, 48, 25-32.

61 H. Sharma, E. Carmichael, M. Muhamad, D. McCall, F. Andrews, G. Lyons, W. McRoberts and P. Hornsby, RSC Adv., 2012, 2, 6424-6437.

62 Q. Zhu, Y. Wang, M. Li, K. Liu, C. Hu, K. Yan, G. Sun and D. Wang, Sep. Purif. Technol., 2017, 186, 70-77.

63 M. Yadav, K. Behera, Y.-H. Chang and F.-C. Chiu, Polymers, 2020, 12, 202.

64 Y. Zuo, W. Liu, J. Xiao, X. Zhao, Y. Zhu and Y. Wu, Int. J. Biol. Macromol., 2017, 103, 1257-1264.

65 A. D. French, Cellulose, 2014, 21, 885-896.

66 J. Gong, J. Li, J. Xu, Z. Xiang and L. Mo, RSC Adv., 2017, 7, 33486-33493.

67 J. W. Rowen, F. H. Forziati and R. E. Reeves, J. Am. Chem. Soc., 1951, 73, 4484-4487.

68 H. Li, B. Wu, C. Mu and W. Lin, Carbohydr. Polym., 2011, 84, 881-886.

69 A. Varma and V. Chavan, Polym. Degrad. Stab., 1995, 49, 245-250.

70 C. D. Tran and T. M. Mututuvari, ACS Sustainable Chem. Eng., 2016, 4, 1850-1861.

71 Y.-H. Yun, Y.-H. Na and S.-D. Yoon, J. Polym. Environ., 2006, 14, 71-78.

72 Q. Li, Z. Ma, Q. Yue, B. Gao, W. Li and X. Xu, Bioresour. Technol., 2012, 118, 204-209.

73 W. Wang and A. Wang, Carbohydr. Polym., 2010, 80, 1028-1036.

74 S. Kiatkamjornwong, K. Mongkolsawat and M. Sonsuk, Polymer, 2002, 43, 3915-3924.

75 K. Haraguchi and T. Takehisa, Adv. Mater., 2002, 14, 1120-1124.

76 L. S. Lim, I. Ahmad, M. A. S. M. Lazim and M. C. I. M. Amin, AIP Conf. Proc., 2014, 1614, 366-370. 